\title{
DIGITALCOMMONS
}

\section{End Matter}

JMASM Editors

Follow this and additional works at: http://digitalcommons.wayne.edu/jmasm

\section{Recommended Citation}

Editors, JMASM (2007) "End Matter," Journal of Modern Applied Statistical Methods: Vol. 6 : Iss. 1 , Article 35. DOI: $10.22237 /$ jmasm/1177994040

Available at: http://digitalcommons.wayne.edu/jmasm/vol6/iss1/35 


\section{Two Years in the Making...}

\section{Intel ${ }^{\oplus}$ Visual Fortran 8.0}

The next generation of Visual Fortran is here! Intel Visual Fortran 8.0 was developed jointly by Intel and the former DEC/Compaq Fortran engineering team.

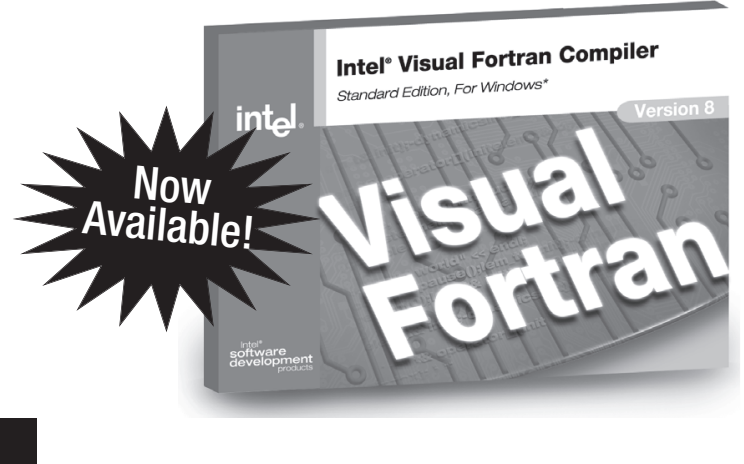

\section{Visual Fortran Timeline}

1997 DEC releases

Digital Visual Fortran 5.0

1998 Compaq acquires DEC and releases DVF 6.0

1999 Compaq ships CVF 6.1

2001 Compaq ships CVF 6.6

2001 Intel acquires CVF engineering team

2003 Intel releases

Intel Visual Fortran 8.0

Intel Visual Fortran $\mathbf{8 . 0}$

- CVF front-end + Intel back-end

- Better performance

- OpenMP Support

- Real*16

\section{Performance}

Outstanding performance on Intel architecture including Intel ${ }^{\circledR}$ Pentium $^{\circledR} 4$, Intel ${ }^{\circledR}$ Xeon $^{\mathrm{TM}}$ and Intel Itanium ${ }^{\circledR} 2$ processors, as well as support for Hyper-Threading Technology.

\section{Compatibility}

- Plugs into Microsoft Visual Studio* .NET

- Microsoft PowerStation4 language and library support

- Strong compatibility with Compaq* Visual Fortran

\section{Support}

1 year of free product upgrades and Intel Premier Support

"The Intel Fortran Compiler 7.0 was first-rate, and Intel Visual Fortran 8.0 is even better. Intel has made a giant leap forward in combining the best features of Compaq Visual Fortran and Intel Fortran. This compiler... continues to be a 'must-have' tool for any Twenty-First Century Fortran migration or software development project."

-Dr. Robert R. Trippi
Professor Computational Finance
University of California, San Diego

FREE trials available at: programmersparadise.com/intel

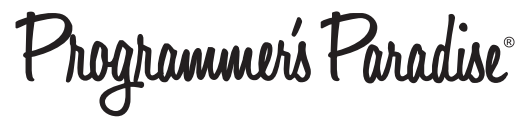

To order or request additional information call: 800-423-9990

Email: intel@programmers.com 
NCSS

329 North 1000 East

Kaysville, Utah 84037

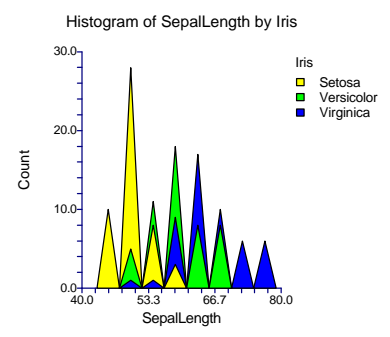

\section{Announcing NCSS 2004 Seventeen New Procedures}

NCSS 2004 is a new edition of our popular statistical NCSS package that adds seventeen new procedures.

\section{New Procedures}

Two Independent Proportions

Two Correlated Proportions

One-Sample Binary Diagnostic Tests

Two-Sample Binary Diagnostic Tests

Paired-Sample Binary Diagnostic Tests

Cluster Sample Binary Diagnostic Tests

Meta-Analysis of Proportions

Meta-Analysis of Correlated Proportions

Meta-Analysis of Means

Meta-Analysis of Hazard Ratios

Curve Fitting

Tolerance Intervals

Comparative Histograms

ROC Curves

Elapsed Time Calculator

T-Test from Means and SD's

Hybrid Appraisal (Feedback) Model

\section{Documentation}

The printed, 330-page manual, called

NCSS User's Guide $V$, is available for

$\$ 29.95$. An electronic (pdf) version of

the manual is included on the distribution

$\mathrm{CD}$ and in the Help system.

\section{Two Proportions}

Several new exact and asymptotic techniques were added for hypothesis testing (null, noninferiority, equivalence) and calculating confidence intervals for the difference, ratio, and odds ratio. Designs may be independent or paired. Methods include: Farrington \& Manning, Gart \& Nam, Conditional \& Unconditional Exact, Wilson's Score, Miettinen \& Nurminen, and Chen.
Meta-Analysis

Procedures for combining studies measuring paired proportions, means, independent proportions, and hazard ratios are available. Plots include the forest plot, radial plot, and L'Abbe plot. Both fixed and random effects models are available for combining the results.

\section{Curve Fitting}

This procedure combines several of our curve fitting programs into one module. It adds many new models such as Michaelis-Menten. It analyzes curves from several groups. It compares fitted models across groups using computerintensive randomization tests. It computes bootstrap confidence intervals.

\section{Tolerance Intervals}

This procedure calculates one and two sided tolerance intervals using both distribution-free (nonparametric) methods and normal distribution (parametric) methods. Tolerance intervals are bounds between which a given percentage of a population falls.

\section{Comparative Histogram}

This procedure displays a comparative histogram created by interspersing or overlaying the individual histograms of two or more groups or variables. This allows the direct comparison of the distributions of several groups.

\section{Random Number Generator} Matsumoto's Mersenne Twister random number generator (cycle length > $10 * * 6000)$ has been implemented.

\section{Binary Diagnostic Tests}

Four new procedures provide the specialized analysis necessary for diagnostic testing with binary outcome data. These provide appropriate specificity and sensitivity output. Four experimental designs can be analyzed including independent or paired groups, comparison with a gold standard, and cluster randomized.

\section{ROC Curves}

This procedure generates both binormal and empirical (nonparametric) ROC curves. It computes comparative measures such as the whole, and partial, area under the ROC curve. It provides statistical tests comparing the AUC's and partial AUC's for paired and independent sample designs.

\section{Hybrid (Feedback) Model}

This new edition of our hybrid appraisal model fitting program includes several new optimization methods for calibrating parameters including a new genetic algorithm. Model specification is easier. Binary variables are automatically generated from class variables.

\section{Statistical Innovations Products}

Through a special arrangement with

Statistical Innovations (S.I.), NCSS

customers will receive $\$ 100$ discounts on:

Latent GOLD ${ }^{\circledR}$ - latent class modeling

SI-CHAID $®$ - segmentation trees

GOLDMineR ${ }^{\circledR}$ - ordinal regression

For demos and other info visit: www.statisticalinnovations.com 
Please rush me the following products:

Qty

NCSS 2004 CD upgrade from NCSS 2001, \$149.95

$\$$

NCSS 2004 User’s Guide V, \$29.95

$\$$

NCSS 2004 CD, upgrade from earlier versions, $\$ 249.95 \ldots \ldots \ldots . . \$$

NCSS 2004 Deluxe (CD and Printed Manuals), \$599.95 ........... \$

PASS 2002 Deluxe, $\$ 499.95$

$\$$

Latent Gold ${ }^{\circledR}$ from S.I., $\$ 995$ - $\$ 100$ NCSS Discount $=\$ 895 \ldots . . . \$$

GoldMineR ${ }^{\circledR}$ from S.I., \$695 - \$100 NCSS Discount $=\$ 595 \ldots . . \$$

CHAID ${ }^{\circledR}$ Plus from S.I., $\$ 695$ - $\$ 100$ NCSS Discount $=\$ 595 \ldots . \$$

Approximate shipping--depends on which manuals are ordered (U.S: \$10 ground, \$18 2-day, or \$33 overnight) (Canada \$24) (All other countries \$10) (Add \$5 U.S. or \$40 International for any S.I. product) ........ \$_

Total...........

CALL: (800) 898-6109 FAX: (801) 546-3907

ONLINE: Www.ncss.com

MAIL: NCSS, 329 North 1000 East, Kaysville, UT 84037

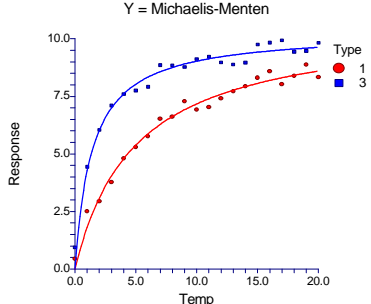

ROC Curve of Fever

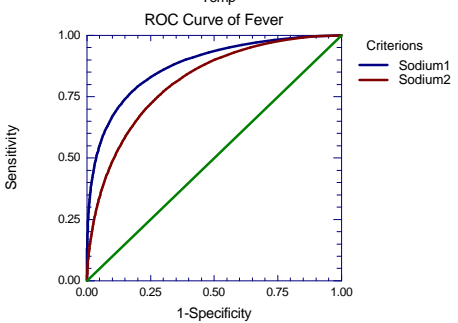

Histogram of SepalLength by lirs

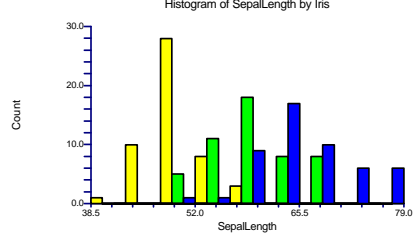

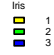

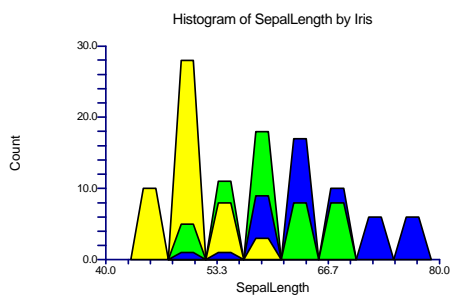

My Payment Option:

Check enclosed

Please charge my: _ VISA _ MasterCard

Amex Purchase order attached

Card Number Exp

Signature

Telephone:

Email:

Ship to:

NAME

ADDRESS

ADDRESS

ADDRESS

CITY STATE

ZIP/POSTAL CODE COUNTRY

\section{Statistical and Graphics Procedures Available in NCSS 2004}

Analysis of Variance / T-Tests Analysis of Covariance

Analysis of Variance

Barlett Variance Test

Crossover Design Analysis

Factorial Design Analysis

Friedman Test

Geiser-Greenhouse Correction

General Linear Models

Mann-Whitney Test

MANOVA

Multiple Comparison Tests

One-Way ANOVA

Paired T-Tests

Power Calculations

Repeated Measures ANOVA

T-Tests - One or Two Groups

T-Tests - From Means \& SD's

Wilcoxon Test

Time Series Analysis

ARIMA / Box - Jenkins

Decomposition

Exponential Smoothing

Harmonic Analysis

Holt - Winters

Seasonal Analysis

Spectral Analysis

Trend Analysis

*New Edition in 2004
Regression / Correlation Survival / Reliability All-Possible Search

Experimental Designs

Balanced Inc. Block

Box-Behnken

Central Composite

D-Optimal Designs

Fractional Factoria

Latin Squares

Placket-Burman

Response Surface

Screening

Taguchi Canonical Correlation Correlation Matrices Cox Regression

Kendall's Tau Correlation Linear Regression Logistic Regression Multiple Regression Nonlinear Regression PC Regression Poisson Regression Response-Surface Ridge Regression Robust Regression Stepwise Regression Spearman Correlation Variable Selection

Accelerated Life Tests

Cox Regression

Cumulative Incidence Exponential Fitting

Extreme-Value Fitting

Hazard Rates

Kaplan-Meier Curves

Life-Table Analysis

Lognormal Fitting

Log-Rank Tests

Probit Analysis

Proportional-Hazards

Reliability Analysis

Survival Distributions

Time Calculator*

Weibull Analysis

Quality Control

Xbar-R Chart

$C, P, N P, U$ Charts

Capability Analysis

Cusum, EWMA Chart

Individuals Chart

Moving Average Chart

Pareto Chart

R \& R Studies

Cluster Analysis

Factor Analysis

Hotelling's T-Squared

Item Analysis

Loglinear Models
Curve Fitting

Bootstrap C.I.'s*

Built-In Models

Group Fitting and Testing

Model Searching

Nonlinear Regression

Randomization Tests*

Ratio of Polynomials

User-Specified Models

Miscellaneous

Area Under Curve

Bootstrapping

Chi-Square Test

Confidence Limits

Cross Tabulation

Data Screening

Fisher's Exact Test

Frequency Distributions

Mantel-Haenszel Test

Correspondence Analysis

Discriminant Analysis

Item Response Analysis

MANOVA

Multi-Way Tables

Multidimensional Scaling

Principal Components
Meta-Analysis* Independent Proportions ${ }^{\star}$ Correlated Proportions* Hazard Ratios* Means*

Binary Diagnostic Tests* One Sample*

Two Samples* Paired Samples Clustered Samples*

Proportions

Tolerance Intervals* Two Independent

Two Correlated ${ }^{*}$

Exact Tests*

Exact Confidence Intervals ${ }^{\star}$

Farrington-Manning ${ }^{\star}$

Fisher Exact Test

Gart-Nam* Method

McNemar Test

Miettinen-Nurminen*

Wilson's Score* Method

Equivalence Tests*

Noninferiority Tests ${ }^{\star}$

Mass Appraisal

Comparables Reports Hybrid (Feedback) Model ${ }^{\star}$ Nonlinear Regression

Sales Ratios 
"Perfection is achieved, not when there is nothing more to add, but when there is nothing left to take away."

- Antoine de Saint Exupery

F is a carefully crafted subset of the most recent version of Fortran, the world's most powerful numeric language.

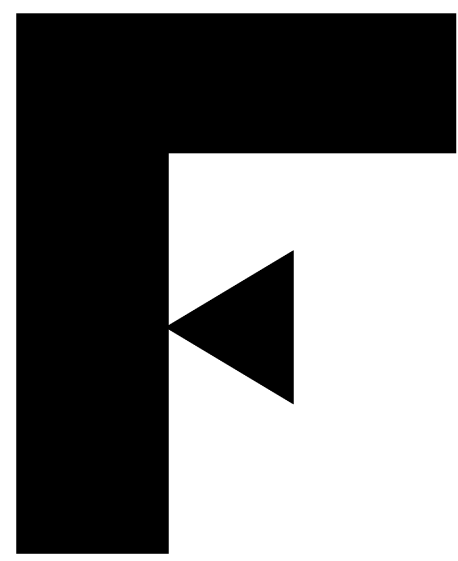

Using F has some very significant advantages:

- Programs written in F will compile with any Fortran compiler

- $\mathrm{F}$ is easier to use than other popular programming languages

- F compilers are free and available for Linux, Windows, and Solaris

- Several books on F are available

- F programs may be linked with C, Fortran 95, or older Fortran 77 programs

F retains the modern features of Fortran-modules and data abstraction, for example-but discards older error-prone facilities of Fortran.

It is a safe and portable programming language.

F encourages Module-Oriented Programming.

It is ideal for teaching a programming language in science, engineering, mathematics, and finance.

It is ideal for new numerically intensive programs.

The Fortran Company

11155 E. Mountain Gate Place, Tucson, AZ 85749 USA $+1-520-256-1455+1-520-760-1397$ (fax) http://www.fortran.com info@fortran.com 


\section{Introducing GGUM2004}

Item Response Theory Models for Unfolding

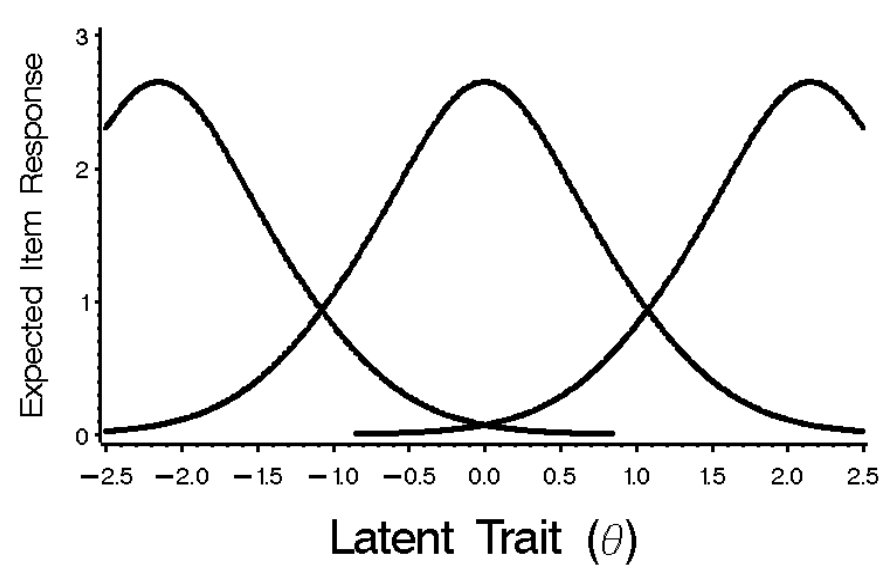

The new GGUM2004 software system estimates parameters in a family of item response theory (IRT) models that unfold polytomous responses to questionnaire items. These models assume that persons and items can be jointly represented as locations on a latent unidimensional continuum. A single-peaked, nonmonotonic response function is the key feature that distinguishes unfolding IRT models from traditional, "cumulative" IRT models. This response function suggests that a higher item score is more likely to the extent that an individual is located close to a given item on the underlying continuum. Such single-peaked functions are appropriate in many situations including attitude measurement with Likert or Thurstone scales, and preference measurement with stimulus rating scales. This family of models can also be used to determine the locations of respondents in particular developmental processes that occur in stages.

The GGUM2004 system estimates item parameters using marginal maximum likelihood, and person parameters are estimated using an expected a posteriori (EAP) technique. The program allows for up to 100 items with 2-10 response categories per item, and up to 2000 respondents. GGUM2004 is compatible with computers running updated versions of Windows 98 SE, Windows 2000, and Windows XP. The software is accompanied by a detailed technical reference manual and a new Windows user's guide. GGUM2004 is free and can be downloaded from:

\section{http://www.education.umd.edu/EDMS/tutorials}

\section{GGUM2004 improves upon its predecessor (GGUM2000) in several important ways:}

- It has a user-friendly graphical interface for running commands and displaying output.

- It offers real-time graphics that characterize the performance of a given model.

- It provides new item fit indices with desirable statistical characteristics.

- It allows for missing item responses assuming the data are missing at random.

- It allows the number of response categories to vary across items.

- It estimates model parameters more quickly.

Start putting the power of unfolding IRT models to work in your attitude and preference measurement endeavors. Download your free copy of GGUM2004 today! 


\section{JOIN DIVISION 5 OF APA!}

The Division of Evaluation, Measurement, and Statistics of the American Psychological Association draws together individuals whose professional activities and/or interests include assessment, evaluation, measurement, and statistics. The disciplinary affiliation of division membership reaches well beyond psychology, includes both members and non-members of APA, and welcomes graduate students.

Benefits of membership include:

- $\quad$ subscription to Psychological Methods or Psychological Assessment (student members, who pay a reduced fee, do not automatically receive a journal, but may do so for an additional $\$ 18$ )

- $\quad$ The Score - the division's quarterly newsletter

- Division's Listservs, which provide an opportunity for substantive discussions as well as the dissemination of important information (e.g., job openings, grant information, workshops)

Cost of membership: $\$ 38$ (APA membership not required); student membership is only $\$ 8$

For further information, please contact the Division's Membership Chair, Yossef Ben-Porath (ybenpora@kent.edu) or check out the Division's website:

http://www.apa.org/divisions/div5/

\section{ARE YOU INTERESTED IN AN ORGANIZATION DEVOTED TO EDUCATIONAL AND BEHAVIORAL STATISTICS?}

Become a member of the Special Interest Group - Educational Statisticians of the American Educational Research Association (SIG-ES of AERA)!

The mission of SIG-ES is to increase the interaction among educational researchers interested in the theory, applications, and teaching of statistics in the social sciences.

Each Spring, as part of the overall AERA annual meeting, there are seven sessions sponsored by SIG-ES devoted to educational statistics and statistics education.

We also publish a twice-yearly electronic newsletter.

Past issues of the SIG-ES newsletter and other information regarding SIG-ES can be found at http://orme.uark.edu/edstatsig.htm

To join SIG-ES you must be a member of AERA. Dues are $\$ 5.00$ per year.

For more information, contact Joan Garfield, President of the SIG-ES, at jbg@umn.edu. 


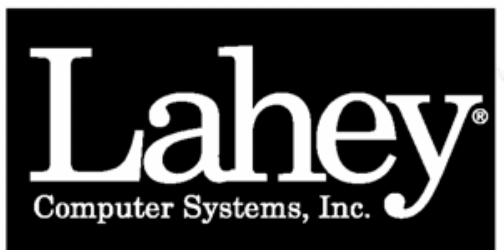

SOFTWARE SOLUTIONS

for Science \& Engineering

\section{Lahey/Fujitsu Fortran}

The standard for Fortran programming from the leader in Fortran language systems

LF95 Fortran for Linux and Windows

Full Fortran 95/90/77 support

Unsurpassed diagnostics

Intel and AMD optimizations
IMSL compatible

Fujitsu SSL2 math library

Wisk graphics package

\section{LF Fortran for the Microsoft ${ }^{\circledR}$.NET Framework - Coming Soon!}

Visual Studio integration

Windows / Web Forms designer

Project and code templates
On-line integrated help

XML Web services

ADO.NET support

Visit www.lahey.com for more information

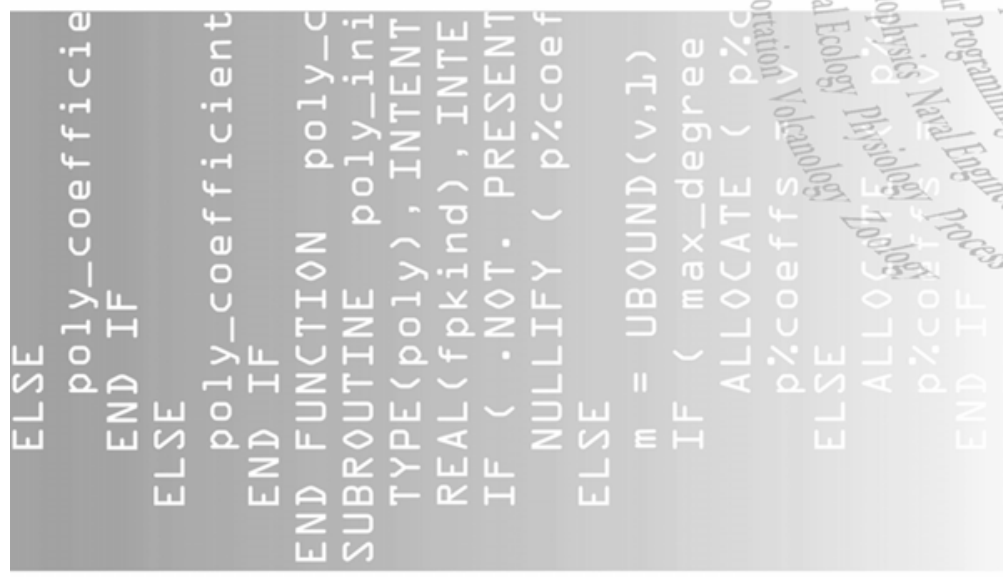

Lahey Computer Systems, Inc.

865 Tahoe Blvd - P.O. Box 6091

Incline Village, NV 89450 USA

1-775-831-2500

www.lahey.com

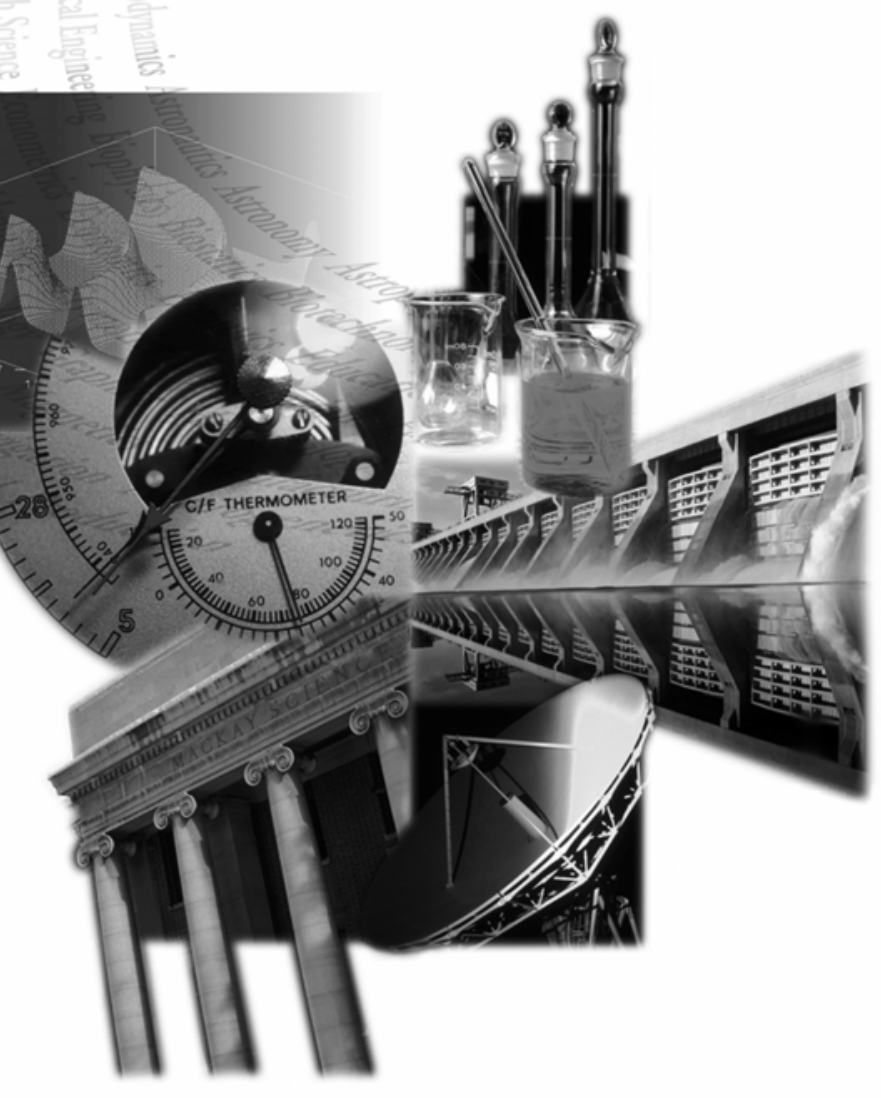




\section{Instructions For Authors}

Follow these guidelines when submitting a manuscript:

1. JMASM uses a modified American Psychological Association style guideline.

2. Submissions are accepted via e-mail only. Send them to the Editorial Assistant at ea@edstat.coe.wayne.edu. Provide name, affiliation, address, e-mail address, and 30 word biographical statements for all authors in the body of the email message.

3. There should be no material identifying authorship except on the title page. A statement should be included in the body of the e-mail that, where applicable, indicating proper human subjects protocols were followed, including informed consent. A statement should be included in the body of the e-mail indicating the manuscript is not under consideration at another journal.

4. Provide the manuscript as an external e-mail attachment in MS Word for the PC format only. (Wordperfect and .rtf formats may be acceptable - please inquire.) Please note that Tex (in its various versions), Exp, and Adobe .pdf formats are designed to produce the final presentation of text. They are not amenable to the editing process, and are NOT acceptable for manuscript submission.

5. The text maximum is 20 pages double spaced, not including tables, figures, graphs, and references. Use 11 point Times Roman font.

6. Create tables without boxes or vertical lines. Place tables, figures, and graphs "in-line", not at the end of the manuscript. Figures may be in .jpg, .tif, .png, and other formats readable by Adobe Illustrator or Photoshop.

7. The manuscript should contain an Abstract with a 50 word maximum, following by a list of key words or phrases. Major headings are Introduction, Methodology, Results, Conclusion, and References. Center headings. Subheadings are left justified; capitalize only the first letter of each word. Sub-subheadings are leftjustified, indent optional.

8. Do not use underlining in the manuscript. Do not use bold, except for (a) matrices, or (b) emphasis within a table, figure, or graph. Do not number sections. Number all formulas, tables, figures, and graphs, but do not use italics, bold, or underline. Do not number references. Do not use footnotes or endnotes.

9. In the References section, do not put quotation marks around titles of articles or books. Capitalize only the first letter of books. Italicize journal or book titles, and volume numbers. Use "\&" instead of "and" in multiple author listings.

10. Suggestions for style: Instead of "I drew a sample of 40" write "A sample of 40 was selected". Use "although" instead of "while", unless the meaning is "at the same time". Use "because" instead of "since", unless the meaning is "after". Instead of "Smith (1990) notes" write "Smith (1990) noted". Do not strike spacebar twice after a period.

\section{Print Subscriptions}

Print subscriptions including postage for professionals are US \$95 per year; for graduate students are US $\$ 47.50$ per year; and for libraries, universities, and corporations are US \$195 per year. Subscribers outside of the US and Canada pay a US $\$ 10$ surcharge for additional postage. Online access is currently free at http://tbf.coe.wayne.edu/jmasm. Mail subscription requests with remittances to JMASM, P. O. Box 48023, Oak Park, MI, 48237. Email journal correspondence, other than manuscript submissions, to jmasm@edstat.coe.wayne.edu.

\section{Notice To Advertisers}

Send requests for advertising information to jmasm@edstat.coe.wayne.edu. 


\section{STATISTICIANS}

\section{HAVE YOU VISITED THE \\ Mathematics Genealogy Project?}

The Mathematics Genealogy Project is an ongoing research project tracing the intellectual history of all the mathematical arts and sciences through an individual's Ph.D. advisor and Ph.D. students. Currently we have over 80,000 records in our database. We welcome and encourage all statisticians to join us in this endeavor.

\section{Please visit our web site}

\section{http://genealogy.math.ndsu.nodak.edu}

The information which we collect is the following:

The full name of the individual, the school where he/she earned a Ph.D., the year of the degree, the title of the dissertation, and, MOST IMPORTANTLY, the full name of the advisor(s). E.g., Fuller, Wayne Arthur; Iowa State University; 1959; A Non-Static Model of the Beef and Pork Economy; Shepherd, Geoffrey Seddon

For additions or corrections for one or two people a link is available on the site. For contributions of large sets of names, e.g., all graduates of a given university, it is better to send the data in a text file or an MS Word file or an MS Excel file, etc. Send such information to:

\section{harry.coonce@ndsu.nodak.edu}

The genealogy project is a not-for-profit endeavor supported by donations from individuals and sales of posters and t-shirts. If you would like to help this cause please send your tax-deductible contribution to: Mathematics Genealogy Project, 300 Minard Hall, P. O. Box 5075, Fargo, North Dakota 58105-5075E 


\section{The easy way to find open access journals}

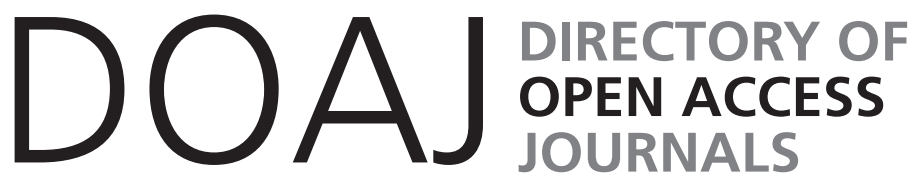

\section{www.doaj.org}

The Directory of Open Access Journals covers free, full text, quality controlled scientific and scholarly journals. It aims to cover all subjects and languages.
Aims
- Increase visibility of open access journals
- Simplify use
- Promote increased usage leading to higher impact

\section{Scope}

The Directory aims to be comprehensive and cover all open access scientific and scholarly journals that use a quality control system to guarantee the content. All subject areas and languages will be covered.

\section{In DOAJ browse by subject}

Agriculture and Food Sciences

Biology and Life Sciences

Chemistry

General Works

History and Archaeology

Law and Political Science

Philosophy and Religion

Social Sciences
Arts and Architecture
Business and Economics
Earth and Environmental Sciences
Health Sciences
Languages and Literatures
Mathematics and statistics
Physics and Astronomy
Technology and Engineering

Contact

Lotte Jørgensen, Project Coordinator

Lund University Libraries, Head Office

E-mail: lotte.jorgensen@lub.lu.se

Tel: +46462223431

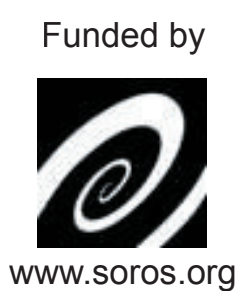

Hosted by

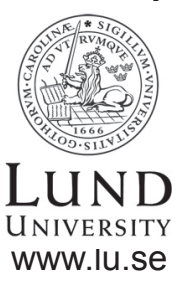

